\title{
Management of foot and ankle injuries in pediatric and adolescent athletes: a narrative review
}

This article was published in the following Dove Press journal:

Orthopedic Research and Reviews

\author{
Laura E Gill',2 \\ Kevin E Klingele ${ }^{1,2}$ \\ 'Department of Orthopedic Surgery, \\ Nationwide Children's Hospital, \\ Columbus, OH, USA; ${ }^{2}$ Department \\ of Orthopaedics, The Ohio State \\ University Wexner Medical Center, \\ Columbus, $\mathrm{OH}$, USA
}

\begin{abstract}
In this review, we focus on the treatment of injuries to the foot and ankle in the adolescent athlete. While many injuries in the adolescent foot and ankle are similar to or overlap with their counterparts in the adult population, the anatomy of the adolescent ankle, especially the presence of growth plates, results in different injury patterns in many cases and calls for specific management approaches. We discuss the unique anatomy of the pediatric patient as well as the diagnostic evaluation and treatment of common injuries in the young athlete.
\end{abstract}

Keywords: foot, ankle, lower extremity, pediatric athlete, growth plate, Lisfranc injury

\section{Introduction}

The foot and ankle are among the most commonly injured regions of the lower extremity in the adolescent athlete. Because of the presence of growth plates, injury patterns among adolescent athletes are different from those in adults, and these injuries require specific management, both non-operative and operative. In some injury patterns there is overlap with adult counterparts; however, treatment may sometimes differ depending on age and skeletal maturity. In these cases, for example, open reduction and internal fixation with physeal sparing or joint preservation techniques, rather than fusion, may be employed. We will review the anatomy of the adolescent ankle joint and focus on the treatment of specific injuries, including os trigonum syndrome, talar osteochondral injuries, tarsal coalitions, Lisfranc injuries, fractures of the base of the fifth metatarsal (MT), syndesmotic injuries, ankle sprains and fractures, and fractures of the great toe.

\section{Anatomy}

The ankle joint is a true hinged joint made up of the articulation of the talus, medial malleolus, distal tibia plafond, and distal fibula/lateral malleolus, along with supporting ligamentous structures. Medially, the ankle joint is stabilized by the deltoid ligament, made up of superficial attachments to the medial malleolus proximally and to the talus, calcaneus, and navicular distally, and deep fibers that insert distally on to the talus. Laterally, there are three ankle-stabilizing ligaments: the anterior and posterior talofibular ligaments (ATFL and PTFL) and the calcaneofibular ligament (CFL). Injuries to the interosseous ligaments, anterior and posterior inferior tibiofibular ligaments that stabilize the ankle mortise, as well as pattern of closure of the distal tibia physis, are important and form the basis of syndesmotic injuries and fractures specific to the adolescent (Tillaux and triplane ankle fractures). ${ }^{1}$
Correspondence: Kevin E Klingele Department of Orthopedic Surgery, Nationwide Children's Hospital, 700 Children's Drive Suite A2700, Columbus, $\mathrm{OH}$ 43205-2696, USA

Tel + I 614 7225175

Fax + I 6I42934755

Email Kevin.Klingele@

nationwidechildrens.org 
The tarsometatarsal (TMT) joint, also described as the Lisfranc joint, is susceptible to injury. It is made up of the articulation of the medial three MTs and their respective cuneiforms and the articulation of the fourth and fifth MTs with the cuboid. The joint is stabilized by the unique bony architecture of the trapezoidal shape of the MTs forming a "Roman arch," with the base of the second MT being the keystone recessed between the medial and lateral cuneiform. The joint is stabilized by transverse ligaments from the second to fifth MTs. The oblique ligament between the medial cuneiform and the base of the second MT is made up of dorsal, interosseous, and plantar ligaments (Lisfranc ligament), of which the interosseous and plantar ligaments are the strongest.

Accessory ossicles in the foot are common in skeletally mature individuals and may be present in up to $20 \%$ of children around the foot and ankle, ${ }^{2}$ including the os trigonum and the accessory navicular, which may become symptomatic.

Ossicles (appearing between 7 and 10 years of age) may be seen inferior to the malleoli. These are commonly bilateral and usually fuse with the secondary ossification center at skeletal maturity, but may persist. The "os subtibiale" is more common and is present in approximately $20 \%$ of individuals, while the "os fibulare" is present in only about $1 \%$ of individuals. ${ }^{3}$

The apophysis of the fifth MT base appears on plain radiographs at the age of 12 years for boys and 10 years for girls. Fusion of the apophysis to the MT base usually occurs within the following 2-4 years. ${ }^{4}$

Developmental changes of the calcaneal apophysis include the onset of ossification at an average age of 8 years in boys and 6 years in girls.

\section{Os trigonum syndrome}

The normal talus has medial and lateral posterior tubercles in which secondary ossification centers appear around 8-10 years of age in girls and 11-13 years in boys and fuse within the same year. ${ }^{5}$ Between these tubercles lies the tendon of the flexor hallucis longus (FHL). Acute fracture or chronic repetitive microtrauma, ${ }^{6}$ lengthening of the lateral tubercle (Steida process), or failure of fusion of the lateral process may result in this painful syndrome owing to impingement of the posterior aspect of the talus and calcaneus. ${ }^{7}$ There is often concomitant FHL pathology with tenosynovitis/hypertrophy of the tendon. Stenosing FHL tendonitis may result in mechanical triggering or locking of the great toe interphalangeal joint. ${ }^{8}$ Athletes, such as dancers, soccer players, divers, and fast bowlers in cricket, require repetitive ankle plantarflexion and may present with restriction in motion, activity-related chronic pain, and swelling to the posterior aspect of the ankle. ${ }^{69}$ Diagnosis is usually made with plain radiographs and computed tomography (CT) (Figure 1), and maximum plantar flexion films may show impingement. Magnetic resonance imaging (MRI) will show inflammation and tenosynovitis and concomitant pathology.

Treatment is initially conservative, with activity modification or rest, ice, non-steroidal anti-inflammatory drugs (NSAIDs), or immobilization in a walker boot or cast for more severe cases. Physical therapy focusing on stretches, strengthening, and proprioception is also implemented. ${ }^{6,10}$ Ultrasound-guided steroid injections may be beneficial and can be both diagnostic and therapeutic. ${ }^{11}$

Surgical treatment is reserved for patients who fail conservative measures and includes open or endoscopic debridement of FHL synovitis and excision of the os trigonum. Release of the FHL sheath may be performed in cases of mechanical symptoms or triggering. ${ }^{8}$

\section{Talar osteochondral injuries}

Osteochondral lesions of the talar dome are defined as a separation of cartilage and subchondral bone secondary to trauma or vascular insult. ${ }^{12}$ They may result from acute trauma or chronic repetitive microtrauma. Ischemia of the subchondral bone causes separation of the fragment from the underlying bed. Osteochondral lesions of the talus occur anterolaterally, but posteromedial lesions are more common and account for

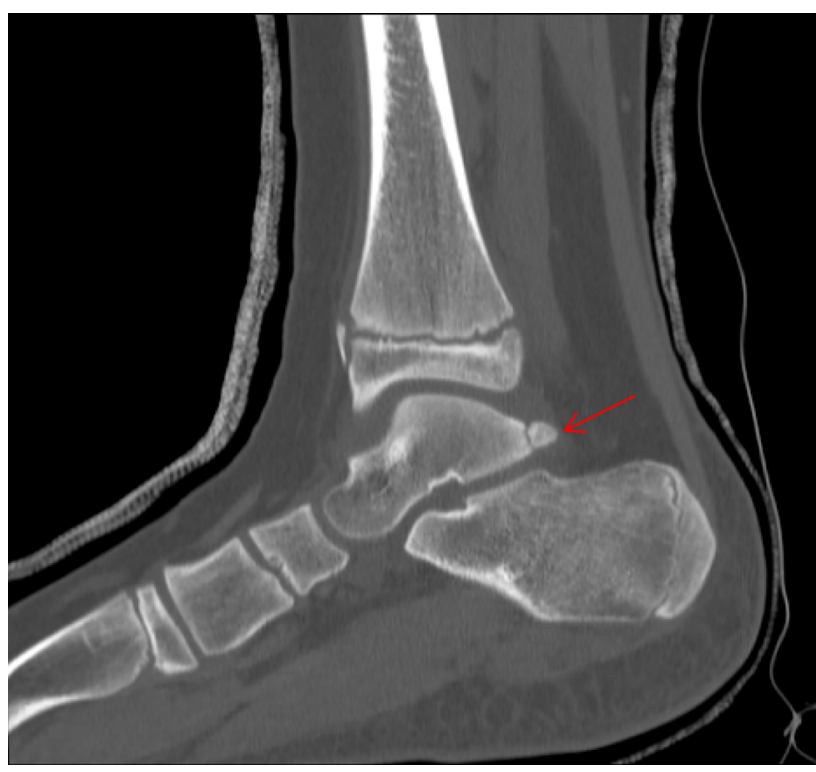

Figure I Lateral view of the ankle, with arrow indicating the os trigonum. 
$74 \%$ of these lesions. ${ }^{13}$ Central lesions are rare. They may be bilateral in $10-25 \%$ of cases. Anterolateral lesions usually result from acute trauma (98\%); these tend to be shallower in depth and larger in size, while posteromedial (PM) lesions usually result from chronic/remote injuries that may be found incidentally on X-ray of the ankle. They are associated with trauma in up to $70 \%$ of cases. PM lesions are smaller in size and deeper in nature.

Patients typically present with pain and swelling of the ankle, but may have feeling of instability or mechanical symptoms such as clicking or locking from a detached or intra-articular loose body. The mechanism of injury for anterolateral lesions is inversion/dorsiflexion force, while PM lesions occur with plantarflexion and inversion.

Diagnosis can typically be made on plain radiographs, with anteroposterior (AP), lateral, and mortise views of the ankle (Figure 2A, B). A CT scan may be done to localize and characterize the lesion, to look for intra-articular loose bodies, and for preoperative planning. MRI is helpful to assess stability and classify the lesion, as well as to assess the integrity of the overlying cartilage (Figure 2C, D). It is also important for diagnosing concomitant injuries of the surrounding ligaments and tendons that may need to be addressed at the same time.

Treatment is based on the staging of the lesion. Berndt and Harty ${ }^{14}$ classified these lesions into four stages: stage 1, small area of subchondral compression; stage 2, partial fragment detachment but non-displaced; stage 3, complete fragment detachment but non-displaced; and stage 4, displaced fragment. Successful non-operative management with NSAIDs, activity modification, or immobilization in a non-weight-bearing splint or cast for 4-6 weeks may be instituted for stage 1 and 2 and small stage 3 lesions. ${ }^{15}$ This is usually followed by progressive weight bearing in a walker boot and physical therapy. Tol et al, ${ }^{16}$ Verhagen et al, ${ }^{17}$ and Letts et al, ${ }^{18}$ in reviewing the literature, showed a $45-54 \%$ success rate for conservative treatment.

Indications for surgical treatment are an extensive period of failed non-operative treatment in symptomatic stage 1-3 lesions and all stage 4 lesions. ${ }^{15}$ Surgery may be performed either arthroscopically or as an open procedure. Anterolateral lesions may be accessed through an anterolateral approach to the ankle, whereas posteromedial lesions may be approached by posteromedial arthrotomy in the interval between the tibialis posterior and the tibia. Osteochondral drilling has the best results when performed for lesions in skeletally immature patients ${ }^{19}$ with intact overlying cartilage, minimal sclerotic changes, and fragment stability (stage 1-2). ${ }^{15,20}$ Antegrade drilling can be accomplished through an arthroscopic portal or arthrotomy. Transmalleolar drilling may be used for lesions with difficult access, but this is not ideal for patients with open physes. Alternatively, retrograde drilling with a targeting guide may be utilized; this has the advantage of not disrupting the intact cartilage. ${ }^{15,21,22}$ For stage 3 and 4 lesions with disrupted cartilage, curettage with excision of underlying necrotic bone and microfracture or drilling of the subchondral bone have shown good results, with formation of fibrocartilage in the defect ${ }^{15,21,22}$ (Figure 2E, F).

Fixation of the fragment should be considered in large fragments $(>1.5 \mathrm{~cm})$, those with acute injuries with no significant underlying sclerosis, and when an osseous component to the affected cartilage is needed to achieve bone fixation.
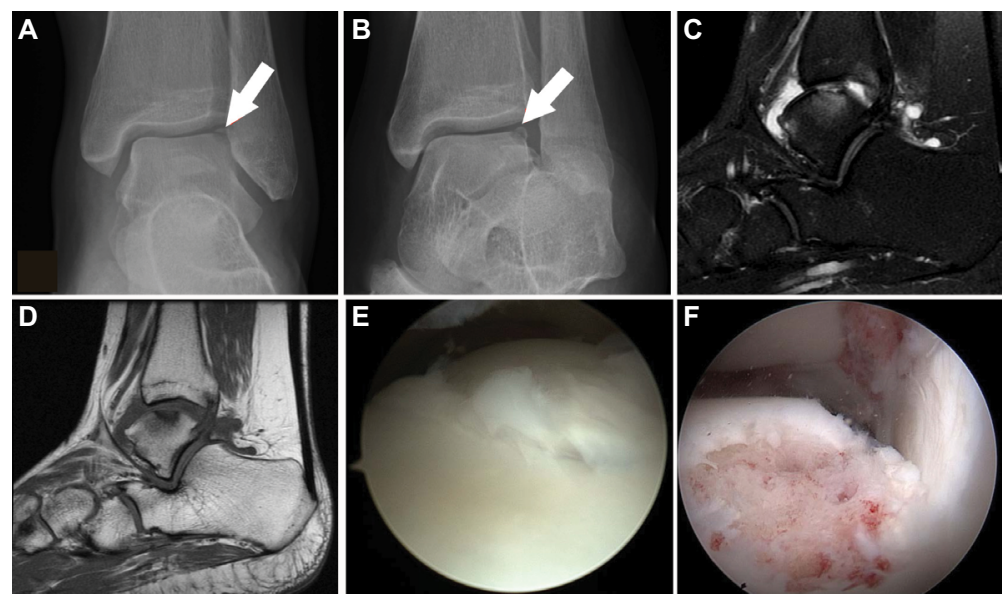

Figure 2 (A) Anteroposterior and (B) mortise views of the ankle demonstrating a posteromedial osteochondral lesion of the talus (arrows); (C,D) magnetic resonance imaging of the lesion showing edema of the underlying talus and fluid beneath the fragment; (E) intraoperative picture of unstable osteochondral lesion; (F) following curettage and microfracture. 
This can be achieved with absorbable pegs or darts, or headless compression screws. These procedures are typically performed with open arthrotomy. Kumai et $\mathrm{al}^{20}$ reported an $89 \%$ success rate in 24 patients with elevation and subchondral drilling of stage 2-4 lesions.

\section{Lisfranc injuries}

Lisfranc injuries are rare in children and adolescents, and are commonly misdiagnosed or go unnoticed. ${ }^{23,24} \mathrm{~A}$ high index of suspicion is necessary in subtle cases or in cases of spontaneous reduction. ${ }^{25}$ Widening of the Lisfranc joint may only be appreciated on stress radiographs, therefore symptomatic patients with a suggestive mechanism of injury or clinical examination should be investigated. A concomitant fracture of the cuboid and base of the second MT is highly suspicious of an injury to the TMT joint. ${ }^{25}$ The mechanism of injury may be direct (e.g. crush injuries) or more commonly indirect from a forced plantarflexion and abduction injury in a fixed foot (e.g. in sports such as football or soccer where another player lands on the heel of the plantarflexed foot, or in a fall from a height). Patients usually present with pain, swelling, and inability to bear weight, and may have plantar ecchymosis in the medial longitudinal arch. With severe swelling, they are at risk of compartment syndrome. ${ }^{25,26}$

Quenu and Kuss ${ }^{27}$ proposed a classification in 1909 based on the direction of displacement and divided these injuries into three categories: homolateral, isolated, and divergent. Myerson et $\mathrm{al}^{28}$ further classified these into types A-C, with subclassification based on the degree of displacement. Hill et al, ${ }^{23}$ in their study, found that $52 \%$ of skeletally immature patients had Myerson type B1 with partial incongruity of the first MT with medial displacement, and $56 \%$ of patients with closed physes had Myerson type B2 with partial incongruity and lateral displacement.

The diagnosis is usually made based on plain AP, lateral, and oblique radiographs (Figure 3A). For subtle injuries, bilateral AP and lateral radiographs may be performed for comparison. MRI may be useful in purely ligamentous injuries to assess the integrity of the Lisfranc ligament. CT scans may be important for preoperative planning, especially in the case of intra-articular injuries and comminution.

Non-operative management may be utilized in minimally displaced injuries ( $<2 \mathrm{~mm}$ displacement) and comprises a non-weight-bearing cast for 6 weeks followed by protected weight bearing. Follow-up radiographs are done in 1-2 weeks to ensure no further displacement. ${ }^{25,26}$

Operative treatment is indicated for more displaced fractures. Closed reduction using finger traps may be used

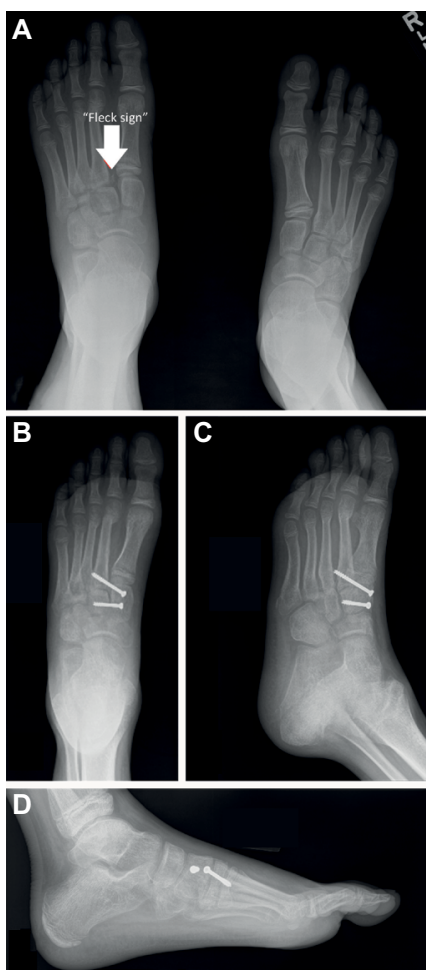

Figure 3 (A) Anteroposterior radiographs of bilateral feet demonstrating widening of the first to second intermetatarsal space, with a positive "fleck sign" representing an avulsion fragment of the ligament from the medial cuneiform. (B) Anteroposterior, (C) oblique, and (D) lateral views of the foot postoperatively, showing reduction and fixation with partially threaded cannulated screws. The patient also had minimally displaced fractures of the second and third metatarsals that were treated non-operatively.

acutely in cases of severe displacement with skin compromise, followed by immediate or delayed treatment depending on the degree of swelling. Staged treatment after a reduction in skin and soft tissue swelling will decrease the risk of skin compromise and concerns with wound healing. Closed treatment with percutaneous K-wire or screw fixation may be an option, ${ }^{29}$ especially in children and adolescents if anatomic or acceptable reduction can be achieved. In older adolescents, open treatment with anatomic direct reduction and fixation with screw fixation is likely to result in better outcomes $^{30,31}$ (Figure 3B-D). In cases in which there is significant comminution of the base of the MTs, bridge plating to the cuneiforms may be necessary. An incision centered between the first and second MTs allows visualization of the respective TMT joints. An incision centered between the third and fourth MTs and a lateral incision are made to address any residual displacement of the fifth TMT joint, as indicated. Rigid fixation of the more rigid medial and middle column of the foot is needed, but with the mobility of the lateral column, fixation with $\mathrm{K}$-wires to the fourth and fifth TMT joints is recommended, with removal of these wires at 
4-6 weeks. Postoperatively, the patient is kept non-weight bearing in a cast or boot for 6-8 weeks, with return to full activities at 3-4 months. Removal of hardware to the medial column is recommended at 3-4 months to allow mobility and prevent screw breakage.

There is a paucity of studies on the management of pediatric Lisfranc injuries, with most of the information gleaned from the adult literature. In the study by Hill et al, ${ }^{23} 56$ patients with bony/ligamentous Lisfranc injuries were reviewed. They found that patients who were skeletally mature were more likely to require surgery. The operative technique employed in $89 \%$ of patients was open reduction and screw fixation. A large proportion of these patients, including 33\% of patients with open physes, required surgery. Long-term outcomes, such as the incidence of post-traumatic arthritis, could not be elucidated in this study; however, the authors' preferred method is joint preservation rather than fusion because of the age and skeletal maturity of these patients, in contrast to the adult population where primary fusion is an option. ${ }^{32,33}$

\section{Fractures of the base of the fifth metatarsal}

Approximately $40 \%$ of all MT fractures occur in the fifth $\mathrm{MT},{ }^{34}$ with up to $22 \%$ involving the base of the MT, of which $90 \%$ occur in children older than 10 years. ${ }^{35}$ The mechanisms of injury include sports, of which soccer is the most common; fall from a height; or a twisting injury of the ankle with a fixed forefoot. ${ }^{36}$ Patients typically present with a history of a twisting/inversion ankle injury with pain and swelling localized to the base of the fifth MT.

Standard AP, lateral, and oblique views of the foot may be used to make the diagnosis; however, consideration of an additional lateral ankle view including the fifth MT may be considered, as up to $23 \%$ of avulsion fractures may be missed on conventional radiographic views. ${ }^{37}$ Lawrence and Botte ${ }^{38}$ divided proximal fractures into three anatomic subgroups: tuberosity avulsion fractures in zone 1 (Figure 4A), fractures at the metaphyseal/diaphyseal junction without extension past the fourth or fifth intermetatarsal articulation (Jones fracture) in zone 2 (Figure 4B), and proximal diaphyseal stress fractures in zone 3 . Torg et $\mathrm{al}^{39}$ further classified Jones fractures based on their potential for healing according to the presence and degree of sclerosis at the fracture site, with type 1 being an acute fracture, type 2 delayed union, and type 3 non-union. These classification systems have implications for treatment based on their potential for healing.

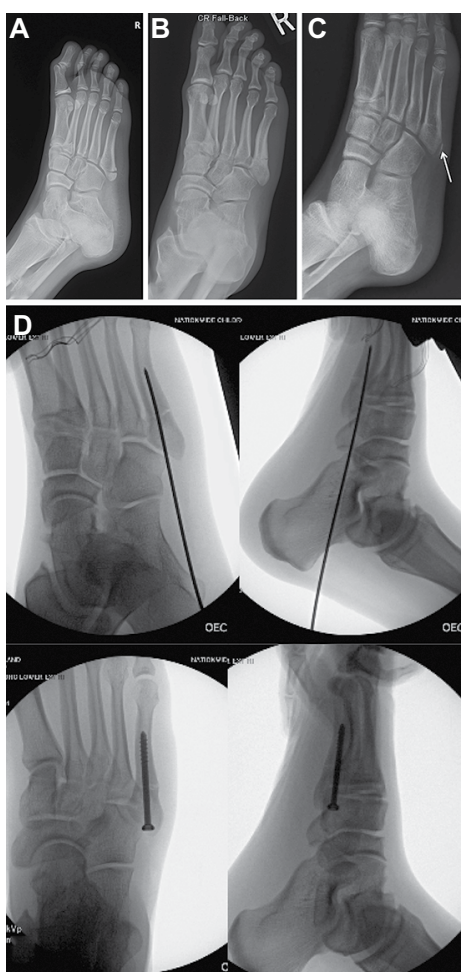

Figure 4 (A) Tuberosity avulsion fractures in zone I; (B) a Jones fracture in zone 2; (C) apophysis of fifth metatarsal, often confused with fracture; (D) clockwise from top left: placement of a guidewire and then a cannulated partially threaded screw for fixation of the Jones fracture.

Avulsion fracture of the base of the fifth MT is the most common isolated injury occurring from an inversion or adduction force, ${ }^{25}$ and is commonly confused with os peroneum and os versalianum or apophysis of the base of the MT (Figure 4C). The injury occurs as a result of a traction pull of the peroneus brevis or origin of the abductor digiti minimi. ${ }^{34}$ Radiographs demonstrate a fracture perpendicular to the plane of the MT and may be intra-articular. They are rarely displaced. Treatment is non-operative, in a hard-soled shoe, short-leg walking cast or boot for 6 weeks. Patients may return to activities when they are pain free with normal strength and range of motion.

For zone 2 and 3 fractures of the fifth MT, displacement greater than 3-4 mm and greater than 10 degrees of angulation should be reduced. ${ }^{40}$ Non-displaced fractures may be given a trial of non-operative treatment with immobilization in a short-leg, non-weight-bearing cast for 6-8 weeks until radiographic union is achieved. ${ }^{40,41}$ Acute fixation should be considered in athletes, as this results in more predictable healing and an earlier return to sport. ${ }^{42,43}$ Intramedullary screw fixation is the method of choice for fixation of these fractures. In cases of delayed union or non-union (Torg 
type 2-3), fixation with or without autologous bone grafting may be employed. A guidewire is passed across the fracture site from a lateral approach at the base of the MT. This wire, once positioned appropriately, may be overdrilled with a cannulated drill to just distal to the fracture site and replaced with a solid screw. The largest screw that will fit within the medullary canal is used; in general, a $4.5 \mathrm{~mm}$ or greater screw is associated with the least risk of refracture or non-union ${ }^{40}$ (Figure 4D).

\section{Tarsal coalitions}

Tarsal coalitions are defined as abnormal connections between two or more bones in the foot. They may be bony (synostosis), fibrous (syndesmosis), or cartilaginous (synchondrosis). ${ }^{44}$ Coalitions are rare, with a prevalence of $1 \%$ of the population and an incidence estimated between $2 \%$ and $13 \% .{ }^{45}$ Up to $50-60 \%$ are bilateral. ${ }^{10,44}$ Talocalcaneal and calcaneonavicular coalitions account for $90 \%$ of the coalitions of the foot. ${ }^{46}$ The middle facet of the calcaneus is most commonly involved.

The restricted motion of the subtalar joint that occurs with tarsal coalitions leads to rigid flat foot deformity. Abnormal loading and stresses across the ankle and subtalar joint result in pain from impingement in the sinus tarsi, peroneal spasm, inflammation, and degenerative joint changes.

Patients typically present at the ages of 8-12 years and 12-16 years for calcaneonavicular and talocalcaneal coalitions, respectively, ${ }^{47}$ as the coalition ossifies and the foot becomes more rigid. Patients presenting with a history of recurrent ankle sprains, especially in the presence of a rigid flat foot deformity, should raise suspicion of a tarsal coalition. The patient may have hindfoot valgus with increased tibiocalcaneal angle that does not correct to varus with heel raise, suggesting rigidity of the subtalar joint. They usually have associated forefoot abduction and may have spasm of the peroneal tendons. The patient may complain of lateral ankle pain in the sinus tarsi as a result of impingement, or directly over the coalition, as in the case of a calcaneonavicular coalition. Patients with talocalcaneal coalition may have medial-sided pain just distal to the medial malleolus. There may be associated contracture of the gastrocsoleus complex and a Silfverskiold test should be performed.

Weight-bearing AP, lateral, and 45 degree non-weightbearing oblique views of bilateral feet should be obtained. On the AP view, there will be uncoverage of the talar head and forefoot abduction. On the lateral view, in talocalcaneal coalitions, there may be a positive $\mathrm{C}$-sign (Figure 5A), which represents a line formed by the medial outline of the dome of the talus and the posteroinferior outline of the sustentaculum tali. ${ }^{48}$ In addition, characteristic beaking of the talar head may be seen, as well as the "anteater nose sign," which represents elongation of the anterior process of the calcaneus towards the navicular in a calcaneonavicular coalition (Figure 6A). A calcaneonavicular coalition is best visualized on the oblique views (Figure 6B). A CT scan can be performed as it shows the bony architecture and can be used to evaluate concomitant coalitions. It can also be used for surgical planning of talocalcaneal coalitions (Figure 5B). MRI scanning is also useful for determining whether there is any additional pathology to soft tissues, tendons, etc.
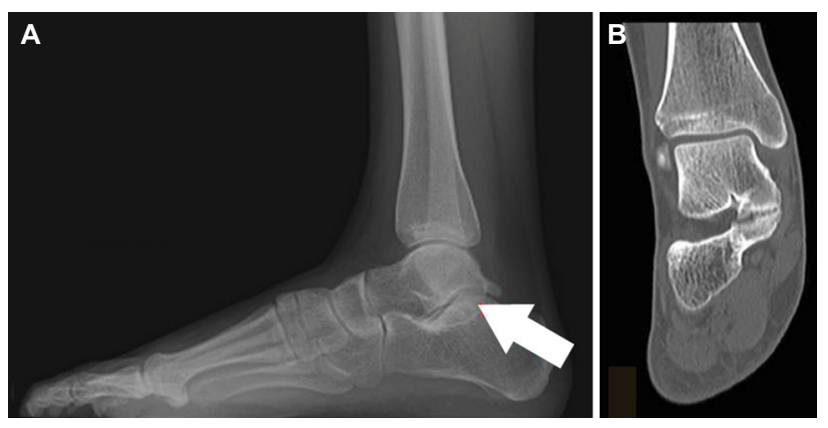

Figure 5 (A) C-sign of talocalcaneal coalition (arrow); (B) coronal computed tomography demonstrating bony coalition.

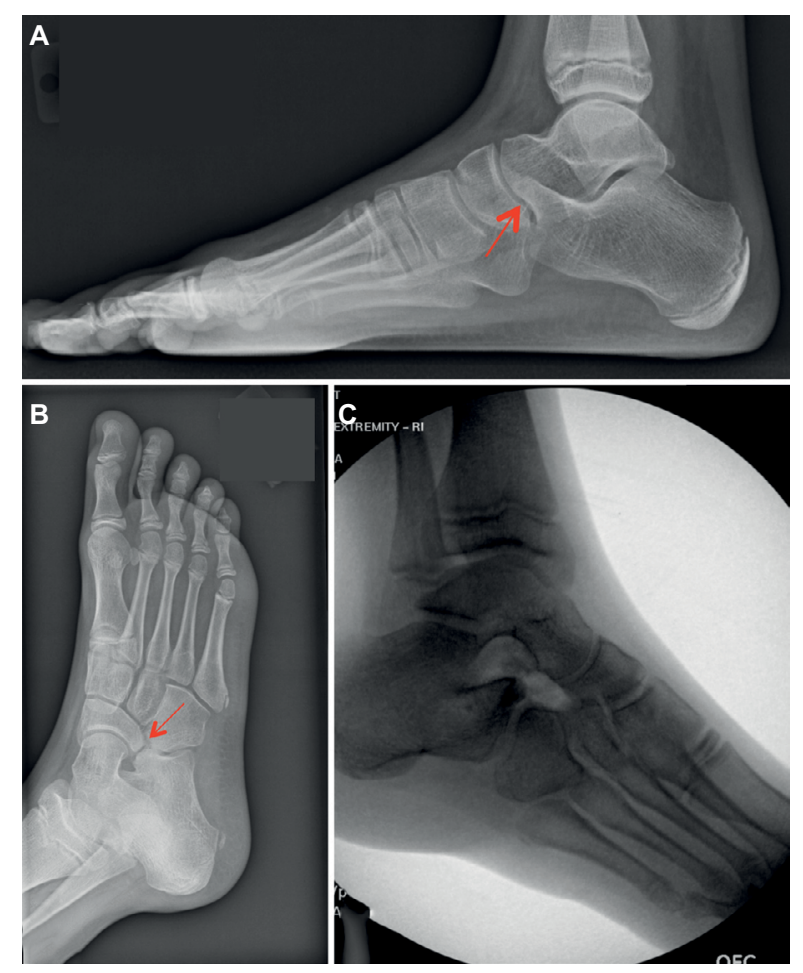

Figure 6 (A) Elongation of the anterior process of the calcaneus (anteater sign, arrow); (B) oblique foot films showing calcaneonavicular coalition (arrow); (C) after resection of the coalition. 
The first line of treatment is non-operative for all coalitions and in asymptomatic patients. It consists of activity modification, analgesics (NSAIDs) or, for more severe cases, immobilization in a walker boot or cast for 4-6 weeks, followed by therapy. Surgical treatment is reserved for patients who have failed non-operative management. For calcaneonavicular coalitions, an Ollier's approach directly over the coalition and resection of a trapezoidal wedge of bone (Figure 6C) with interposition of the extensor digitorum brevis, fat, or bone wax is performed ${ }^{49} \mathrm{Up}$ to $80-90 \%$ acceptable results are achieved. The choice of interposition material is controversial, but it is thought that adequate hemostasis and adequate resection of the coalition are associated with a decreased risk of recurrence and improved outcome. ${ }^{50}$

Talocalcaneal coalitions without associated significant flatfoot deformity may be treated with resection alone if they involve less than $50 \%$ of the middle facet and there are no significant degenerative changes of the posterior facet. ${ }^{51,52}$ This is done via a transverse incision just distal to the medial malleolus and directly over the coalition. A rectangular piece of bone is resected using a combination of osteotomes and burrs, creating a clear space from the posterior to anterior facet, visualizing the cartilage of the subtalar joint medially. The anatomy can be difficult to assess intraoperatively. Aibinder et $\mathrm{al}^{53}$ suggested that the use of intraoperative three-dimensional CT-assisted resection reduces morbidity, with less bone removed and preservation of intact subtalar articulations, and allows for an efficient, thorough, and controlled resection of the coalition. Interposition materials include autologous fat, bone wax, or flexor halluces longus tendon. In cases of flatfoot deformity with severe hindfoot valgus ( $>16$ degrees), several authors have suggested that medial displacement calcaneal/calcaneal lengthening leads to improved outcomes. ${ }^{54,55}$

Postoperative treatment involves immobilization in a splint or cast for 3 weeks, then transition to a walker boot and physical therapy. An over-the-counter orthotic may be used in the long term. The patient can usually return to sport 3-4 months postoperatively.

\section{Ankle sprains \\ Lateral ankle sprains}

The incidence of ankle sprains has been estimated at 2.15 per 1000 person-years in the USA. The peak incidence of ankle sprain occurs between 15 and 19 years of age. Half of all ankle sprains occur during athletic activity, ${ }^{56}$ with lateral ankle sprain and syndesmotic sprain being the most common foot and ankle injuries in collegiate football players, occurring in $31 \%$ and $15 \%$ of players, respectively. ${ }^{57}$ Recurrent ankle sprains may result in chronic instability and pain in the ankle, with loss of normal ankle kinematics and proprioception, placing patients at risk for degenerative changes of the ankle. ${ }^{58,59}$

These sprains usually result from inversion of the foot with associated external rotation of the ankle joint. ${ }^{58}$ The lateral ankle ligaments are injured most commonly. They are comprised of the ATFL, PTFL, and CFL. Of these, the ATFL is most commonly injured, followed by the CFL..$^{57,58}$ In addition, the ankle musculature, specifically the peroneus longus and brevis, actively stabilizes the ankle joint. Weakness or injury, such as tendon tears or subluxation (rupture of the superior peroneal retinaculum), may play a role in ankle instability. ${ }^{57}$

Ankle sprains are classified into three grades based on the degree of ligament injury and laxity of the ankle joint. ${ }^{60}$ Grade I is associated with a stretch of the ligaments without frank tear and no instability. In grade II injuries, there is complete tear of the ATFL with or without partial tear of the ATFL, and there may be mild instability of the joint. Grade III tears are severe, associated with complete tear of the ATFL and CFL, with possible PTFL and capsular injury and ankle instability.

Patients typically present with pain, swelling and bruising to the ankle joint, and difficulty in bearing or inability to bear weight. They may have a history of recurrent ankle sprains or chronic instability. The stability of the ankle joint is assessed using the anterior drawer test; this applies an anteriorly directed force to the hindfoot while stabilizing the tibia and tests the integrity of the ATFL, which is the primary resistance to anterior subluxation of the talus. Testing for the CFL involves tilting the talus with inversion stress testing and the ankle in neutral dorsiflexion.

The need for radiographs is determined based on the Ottawa rules, ${ }^{61}$ and views of the ankle include standard AP, lateral, and mortise to rule out associated fractures. The value of stress radiographs is controversial. ${ }^{57} \mathrm{MRI}$ may be of value in determining the nature and extent of the injury, but should be reserved for chronic injuries that fail to respond to conservative measures and for preoperative planning or to rule out concomitant injuries.

\section{Treatment of acute ankle sprains}

The first line of treatment of an acute ankle sprain is non-operative treatment. ${ }^{60}$ The National Athletic Trainers' Association Position Statement ${ }^{59}$ recommends cryotherapy, NSAIDs, compression, and elevation. Functional rehabilitation is more effective than immobilization for grade I and 
II sprains. For grade III sprains, a short period of immobilization with a rigid brace or cast, followed by controlled rehabilitation, is recommended. Electrical stimulation may be used to minimize swelling in the acute phase.

Preventive treatment of ankle sprain is important in avoiding primary and recurrent injuries and should be aimed at reducing modifiable risk factors, such as body mass index. It includes injury prevention programs, braces or taping, strengthening, reduced participation in sport or change in player position, and modification of playing surfaces and equipment. ${ }^{57}$ Physical therapy with proprioception, single-leg balance training, and neuromuscular control has shown to be the most effective in preventing ankle sprains. ${ }^{57}$ Strength and range of motion should also be initiated simultaneously. Bracing or taping can also be used to stabilize and has been shown to reduce the incidence of recurrent sprains by $70 \%$ in at-risk individuals. ${ }^{62}$

\section{Treatment of chronic ankle sprains with instability}

Surgical treatment is reserved for patients with chronic instability who have failed conservative measures. The approach may be placed into two main categories: anatomic fixation and tenodesis technique. ${ }^{57,60}$ In addition, bony correction of any hindfoot malalignment, e.g. cavovarus foot deformity, should be addressed to prevent failure.

Anatomic fixation with direct repair of the ATFL and CFL was first described by Brostrom and later modified by Gould. ${ }^{63}$ The ruptured or attenuated ligaments are shortened and repaired to their anatomic location on the fibula with suture, suture anchor, or drill holes, and the lateral portion of the inferior extensor retinaculum is brought proximally and sutured to the periosteum of the fibula to add additional stability and reinforce the repair. This repair depends on the integrity of the remaining ligament, and more recently suture tape fixation (InternalBrace ${ }^{\mathrm{TM}}$; Arthrex, Naples, FL, USA) has been used successfully in these situations to augment repair. ${ }^{64,65}$ In patients whose ligaments are attenuated and for whom repair is not feasible, anatomic repair may be performed with other tissue, such as a periosteal flap, a gracilis tendon autograft, or an allograft.

Tenodesis techniques involve the use of either whole or split peroneus brevis tendon weaved and placed through drill holes in the distal fibula, calcaneus, and/or talus in an attempt to re-create and restore ankle stability. The most common techniques described are the Watson-Jones, ChrismanSnook, and Evans procedures.
The purported benefit of anatomic fixation is preservation of more normal ankle mechanics. In general, studies have demonstrated that anatomic repair is associated with better outcomes with fewer complications than tenodesis techniques, and is generally preferred. ${ }^{60,66}$ Anatomic techniques spare the peroneus tendons, which are important for maintaining normal ankle mechanics and muscle balance.

\section{Syndesmotic ankle sprains}

High ankle sprains are injuries occurring to ligaments involving the ankle syndesmosis superior to the more distal ankle ligaments. The ligaments involved are the anterior inferior tibiofibular, posterior inferior tibiofibular, and interosseous ligaments. The deltoid ligament also provides stability as it resists lateral talar shift. These injuries may occur with or without a fibula fracture. The mechanism of injury involves external rotation of the foot with the ankle in dorsiflexion. The talus forces separation of the syndesmosis. It is more common in high-speed sports that involve jumping, cutting, and direction change, such as soccer, basketball, and hockey.,57

Patients typically present with pain and swelling around the ankle joint. Clinical examination may reveal swelling. Several specific tests are available for use in evaluating patients with suspected syndesmotic injuries. The squeeze test involves compression of the tibia and fibula at the level of the midleg, with the patient sitting with knee flexed at 90 degrees off the table. A positive test results if pain is elicited. The external rotation test is performed by externally rotating the foot with stabilization of the tibia. The squeeze test is more sensitive and specific than the external rotation test. ${ }^{67}$

Standard AP, lateral, and mortise views of the ankle joint may be normal or show widening of the medial clear space of the ankle mortise with lateral talar shift (Figure 7A). Imaging should also include full-length tibia and fibula films to evaluate for a concomitant high fibula fracture (Maisonneuve

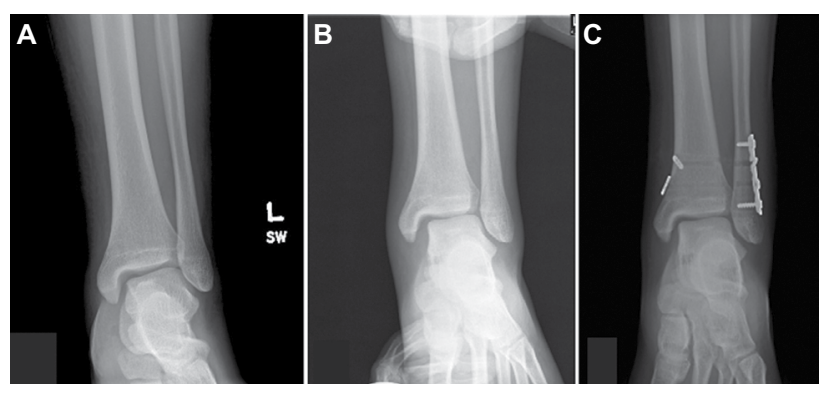

Figure 7 A 16-year-old male sustained an injury playing football. (A) The initial ankle mortise appears normal; (B) however, external rotation stress films demonstrate widening of the mortise and medial clear space with talar shift; (C) after syndesmotic fixation with tightrope construct. 
fracture). Stress radiographs may be necessary to demonstrate the instability at the syndesmosis and should be done in cases with a high index of suspicion. These include bilateral weight-bearing standing AP views of ankles or bilateral external rotation stress radiographs (Figure 7B). Decreased tibiofibular overlap (normal $>6 \mathrm{~mm}$ on AP view, $>1 \mathrm{~mm}$ on mortise view), increased medial clear space (normal $\leq 4 \mathrm{~mm}$ ), or increased tibiofibular clear space (normal $<6 \mathrm{~mm}$ on both AP and mortise views) is suggestive of syndesmotic injury. MRI of the ankle joint is both sensitive and specific in detecting syndesmotic ligaments and any associated injuries. ${ }^{68}$ Syndesmotic injuries are classified as grade I-III, with grades I and II having partial disruption of the ligaments and grade III having complete tear of the ligaments with mortise instability. However, this classification system does not specify treatment or prognostic information. ${ }^{69}$

Treatment of high ankle sprains is based on the stability of the ankle mortise. For injuries without ankle instability, treatment is non-operative and consists of rest, ice, antiinflammatory medications, and immobilization in a walker boot or cast for 3-4 weeks or until the patient is pain free. The patient is then allowed progressive protected weight bearing and rehabilitation with supervised physical therapy, working on proprioception training, neuromuscular training, strengthening, and range of motion. Criteria for return to sport are similar to those for ankle sprains, but the patient should be advised that this generally takes longer than for lateral ankle sprains. ${ }^{57,69}$

For patients with instability on imaging, surgical treatment and syndesmotic fixation with or without fracture fixation is recommended. A lateral approach to the distal fibula is performed; a clamp is placed across the tibia and fibula with the ankle in maximum dorsiflexion to reduce the mortise. Syndesmotic fixation may then be achieved with two parallel 3.5 or $4.5 \mathrm{~mm}$ solid screws placed $2-5 \mathrm{~cm}$ above the ankle joint. Moore et al, in a randomized controlled trial of 120 patients with syndesmotic disruption, found no difference between three- and four-cortex fixation with regard to loss of reduction, screw breakage, or the need for implant removal. ${ }^{70}$ Alternative fixation may be attained with a suture button that consists of a strong suture loop tensioned and secured between two metallic buttons that abut the outer cortices of the tibia and fibula with or without a fibular plate ${ }^{69}$ (Figure 7C). The flexibility of the suture button construct is thought to result in a more anatomic fixation of the syndesmosis as the micromotion allows the fibula to "settle" back into the incisuria of the tibia and reduces the incidence of malreduction; the added advantage is that this device does not routinely require removal.

Inge et $\mathrm{al}^{71}$ performed a systematic review of static versus dynamic syndesmotic fixation and found that dynamic fixation was a viable alternative to the static fixation device, with lower reoperation rates and fewer complications. This approach can accurately stabilize the ruptured syndesmosis without device breakage or loss of reduction.

For athletes with lateral and high ankle sprains, return to play is allowed when the range of motion and strength of the injured extremity have returned to baseline or at least $90 \%$ of the normal side. Athletes with a history of prior ankle sprain should be prophylactically treated with either taping or bracing during participation in sport to prevent reinjury. ${ }^{55}$

\section{Ankle fractures}

Foot and ankle fractures account for $12 \%$ of all pediatric fractures. ${ }^{72}$ Acute fractures in the pediatric population are unique because of the presence of the physis, which acts as an area of weakness, and usually the injury occurs through this region. ${ }^{10}$ The most commonly used classification system is the Salter-Harris ( $\mathrm{SH}$ ) classification. ${ }^{73}$ Type I injuries involve the growth plate without injury to the adjacent bone. Type II injuries involve the physis and a metaphyseal fragment. Type III injuries occur when the fracture line extends from the physis into the epiphysis. Type IV injuries extend from the metaphysis, through the physis, and into the epiphysis in a vertical manner. Type $\mathrm{V}$ injuries involve a compressive or crush injury to the physis. Types I and $\mathrm{V}$ are often not evident on plain radiographs, as only the physis is involved. Types III and IV fractures are intra-articular and are at highest risk of physeal arrest with risk of angular deformity. This risk is up to $38 \%$, especially in the medial malleolar type. ${ }^{74}$ Of these, type IV fractures have the highest risk since they involve all layers of the physis and, as such, these fractures should be anatomically reduced and fixed.

SH type I fracture of the distal fibula is the most common fracture pattern seen in the ankle region. This fracture is the equivalent to the pediatric ankle sprain. It may be difficult to definitively diagnose owing to the lack of initial radiographic findings. Sankar et $\mathrm{al}^{75}$ found that $18 \%$ of patients presenting with pain and swelling around the lateral ankle had an occult fracture observed by periosteal reaction on later films. Typically, patients may have a non-displaced SH type I or II distal fibula fracture or avulsion fracture to the tip of the malleolus. Immobilization in a walker boot or short-leg, weight-bearing cast is appropriate for 3-4 weeks. 
Two unique fracture patterns (juvenile Tillaux and triplane ankle fractures) occur around the distal tibia near skeletal maturity and are related to the pattern in which the distal tibia closes from central to anteromedial, posteromedially, and then laterally over the course of 18 months. ${ }^{76}$

Tillaux ankle fractures are equivalent to a SH type III fracture and involve the anterolateral portion of the ankle joint. The mechanism of injury is a dorsiflexion external rotation force with avulsion of the fragment by the anterior inferior talofibular ligament.

Triplane ankle fractures are three-dimensional and involve the coronal, axial, and sagittal planes. On the AP radiograph of the ankle, a triplane fracture appears as an $\mathrm{SH}$ III fracture, while on the lateral views it appears as an $\mathrm{SH}$ type II fracture with a posteromedial fragment (Figure 8). The mechanism of injury is similar to the Tillaux ankle fracture.
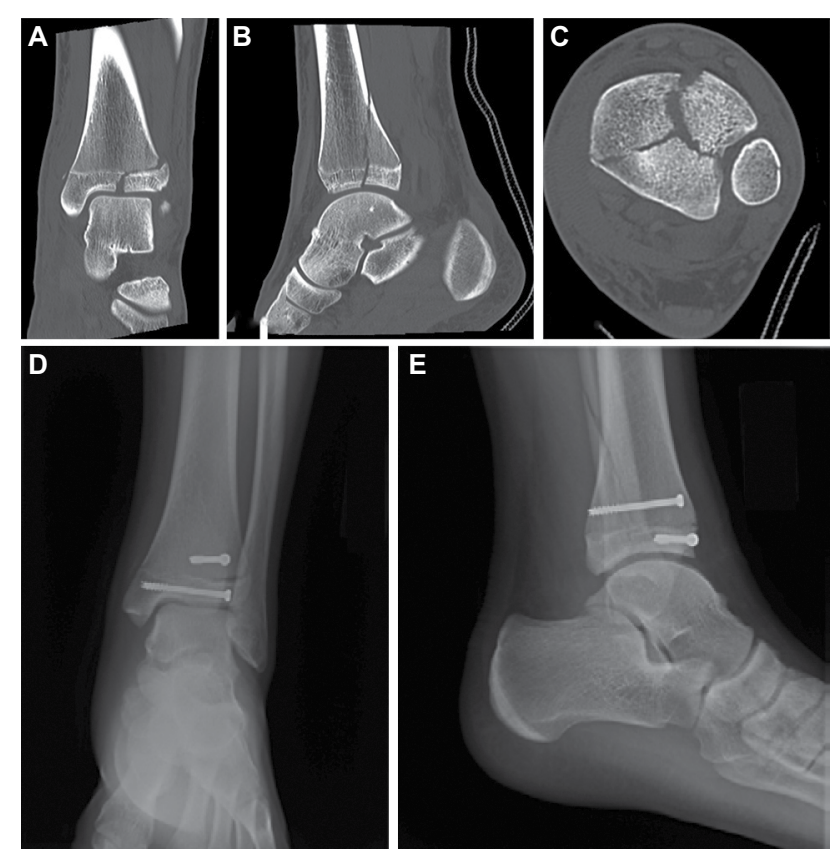

Figure 8 (A-C) Triplane ankle fracture variant with Salter-Harris type IV component (magnetic resonance imaging); (D,E) postoperative fixation with cannulated screws parallel to the physis (D, anteroposterior; E, lateral).
Patients present with pain, swelling, and deformity to the ankle. Non-displaced fractures and fractures with less than $2 \mathrm{~mm}$ displacement at the articular surface after reduction may be managed non-operatively in a long-leg cast for 4 weeks followed by conversion to a non-weight-bearing, short-leg cast or walker boot for a further 2 weeks. Closed reduction is performed under sedation in the emergency room. For Tillaux fractures, the reduction maneuver is plantarflexion and internal rotation to reduce the fracture fragment. A similar internal rotation maneuver is used for the triplane ankle fracture, but in addition, ankle dorsiflexion is typically needed for reduction of the SH II component. CT scans should be performed for all intra-articular fractures after reduction as greater than $2 \mathrm{~mm}$ of displacement of the intra-articular portion is an indication for surgery (Figure 9).

In skeletally immature individuals, fixation using a physeal sparing technique is recommended. Typically, a $3.5-4.0 \mathrm{~mm}$ cannulated screw is placed perpendicular to the fracture site. For SH type IV and triplane fractures, a screw may be required in both the metaphyseal and epiphyseal fragments for fracture stability. The approach depends on the location of the epiphyseal fragment: a lateral approach is used for Tillaux fractures and a medial approach for medial malleolar or triplane fractures. Arthrotomy and direct visualization of the fragment are recommended to ensure an anatomic reduction. Postoperative immobilization is in a short-leg, non-weight-bearing cast for 4-6 weeks.

\section{Great toe phalangeal fractures}

Phalangeal fractures may account for up to $18 \%$ of foot fractures. ${ }^{34}$ Most commonly these are SH type I and II fractures. When this fracture involves the distal phalanx, care must be taken to examine the nailbed for injury, as this qualifies as an open fracture and has a high risk of late osteomyelitis if treated inappropriately. ${ }^{77,78} \mathrm{~A}$ closed reduction of displaced fractures is performed with K-wire stabilization as necessary. Injury to the germinal matrix is suggested by any bleeding
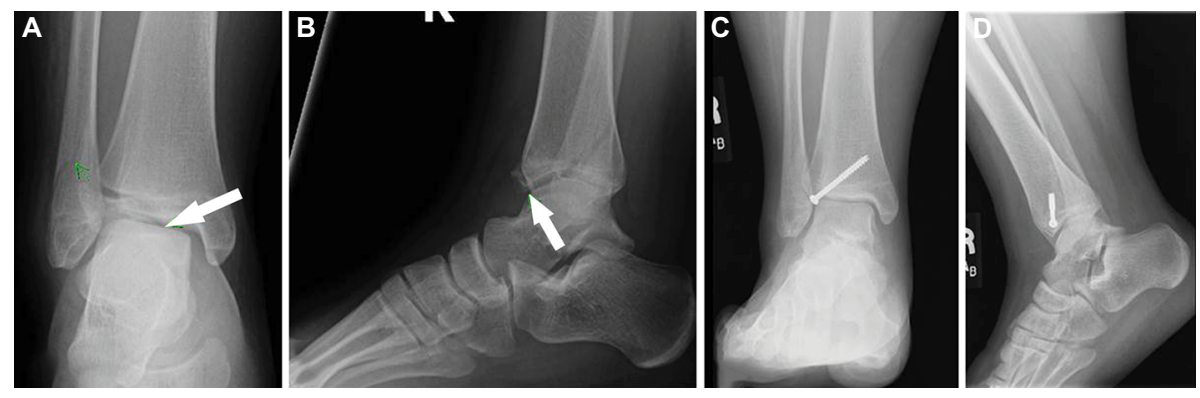

Figure 9 (A) Posterior and (B) lateral Tillaux ankle fracture (arrows); with (C) posterior and (D) transphyseal fixation, in a nearly skeletally mature female. 
around the nail and requires nail removal, irrigation and debridement, and repair. The patient should be treated with a short course of antibiotics, typically a first-generation cephalosporin or clindamycin. Immobilization in a postoperative/ hard-soled shoe for 4 weeks is recommended.

\section{Conclusion}

Because of fundamental anatomic differences, the treatment of foot and ankle injuries among adolescent athletes differs from that in adults. Non-operative management is the first line of treatment in most cases. The acquisition of appropriate radiographic imaging for the suspected injury is key in determining the correct diagnosis and course of treatment. A stepwise approach and appropriate management of the adolescent patient are required to allow return to sport with full and pain-free function. It is important to avoid physeal injury with fixation and consideration should be given to joint preservation techniques wherever possible in this population.

\section{Disclosure}

The authors received no funding for this study, and report no conflicts of interest.

\section{References}

1. Kay RM, Matthys GA. Pediatric ankle fractures: evaluation and treatment. J Am Acad Orthop Surg. 2001;9(4):268-278.

2. Herring JA. Tachdjian's Pediatric Orthopaedics: From the Texas Scottish Rite Hospital for Children. 5th ed. New York: Elsevier Saunders; 2013.

3. Ogden JA, Lee J. Accessory ossification patterns and injuries of the malleoli. J Pediatr Orthop. 1990;10(3):306-316.

4. Canale ST, Williams KD. Iselin's disease. J Pediatr Orthop. 1992;12(1):90-93.

5. Lawson JP. Symptomatic radiographic variants in extremities. Radiology. 1985;157(3):625-631.

6. Lavery KP, McHale KJ, Rossy WH, Theodore G. Ankle impingement. J Orthop Surg Res. 2016;11(1):97.

7. Grogan DP, Walling AK, Ogden JA. Anatomy of the os trigonum. J Pediatr Orthop. 1990;10(5):618-622.

8. Kolettis GJ, Micheli LJ, Klein JD. Release of the flexor hallucis longus tendon in ballet dancers. J Bone Joint Surg Am. 1996;78(9):1386-1390.

9. Nault ML, Kocher MS, Micheli LJ. Os trigonum syndrome. J Am Acad Orthop Surg. 2014;22(9):545-553.

10. Erickson JB, Samora WP, Klingele KE. Ankle injuries in the pediatric athlete. Sports Med Arthrosc. 2016;24(4):170-177.

11. Robinson P, Bollen SR. Posterior ankle impingement in professional soccer players: effectiveness of sonographically guided therapy. AJR Am J Roentgenol. 2006;187(1):W53-W58.

12. Grossman JP, Lyons MC, 2nd. A review of osteochondral lesions of the talus. Clin Podiatr Med Surg. 2009;26(2):205-226.

13. Higuera J, Laguna R, Peral M, Aranda E, Soleto J. Osteochondritis dissecans of the talus during childhood and adolescence. $J$ Pediatr Orthop. 1998;18(3):328-332.

14. Berndt AL, Harty M. Transchondral fractures (osteochondritis dissecans) of the talus. J Bone Joint Surg Am. 1959;41-A:988-1020.

15. Badekas T, Takvorian M, Souras N. Treatment principles for osteochondral lesions in foot and ankle. Int Orthop. 2013;37(9):1697-1706.
16. Tol JL, Struijs PA, Bossuyt PM, Verhagen RA, van Dijk CN. Treatment strategies in osteochondral defects of the talar dome: a systematic review. Foot Ankle Int. 2000;21(2):119-126.

17. Verhagen RA, Struijs PA, Bossuyt PM, van Dijk CN. Systematic review of treatment strategies for osteochondral defects of the talar dome. Foot Ankle Clin. 2003;8(2):233-242, viii-ix.

18. Letts M, Davidson D, Ahmer A. Osteochondritis dissecans of the talus in children. J Pediatr Orthop. 2003;23(5):617-625.

19. Bradley J, Dandy DJ. Results of drilling osteochondritis dissecans before skeletal maturity. J Bone Joint Surg Br. 1989;71(4):642-644.

20. Kumai T, Takakura Y, Higashiyama I, Tamai S. Arthroscopic drilling for the treatment of osteochondral lesions of the talus. J Bone Joint Surg Am. 1999;81(9):1229-1235.

21. Jurina A, Dimnjakovic D, Mustapic M, Smoljanovic T, Bojanic I. Clinical and MRI outcomes after surgical treatment of osteochondral lesions of the talus in skeletally immature children. J Pediatr Orthop. 2018;38(2):122-127.

22. Zengerink M, Struijs PA, Tol JL, van Dijk CN. Treatment of osteochondral lesions of the talus: a systematic review. Knee Surg Sports Traumatol Arthrosc. 2010;18(2):238-246.

23. Hill JF, Heyworth BE, Lierhaus A, Kocher MS, Mahan ST. Lisfranc injuries in children and adolescents. J Pediatr Orthop B. 2017;26(2):159-163.

24. Veijola K, Laine HJ, Pajulo O. Lisfranc injury in adolescents. Eur J Pediatr Surg. 2013;23(4):297-303.

25. Ribbans WJ, Ribbans HA, Cruickshank JA, Wood EV. The management of posterior ankle impingement syndrome in sport: a review. Foot Ankle Surg. 2015;21(1):1-10.

26. Watson TS, Shurnas PS, Denker J. Treatment of Lisfranc joint injury: current concepts. J Am Acad Orthop Surg. 2010;18(12):718-728.

27. Quenu E, Kuss G. Study on the dislocations of the metatarsal bones (tarsometatarsal dislocations) and diastasis between the 1st and 2 nd metatarsals [French]. Rev Chir 1909;39:281-336,720-791, 1093-1134.

28. Myerson MS, Fisher RT, Burgess AR, Kenzora JE. Fracture dislocations of the tarsometatarsal joints: End results correlated with pathology and treatment. Foot Ankle 1986;6(5):225-242.

29. Myerson MS, Cerrato R. Current management of tarsometatarsal injuries in the athlete. Instr Course Lect. 2009;58:583-594.

30. Myerson MS, Fisher RT, Burgess AR, Kenzora JE. Fracture dislocations of the tarsometatarsal joints: end results correlated with pathology and treatment. Foot Ankle. 1986;6(5):225-242.

31. Arntz CT, Hansen ST Jr. Dislocations and fracture dislocations of the tarsometatarsal joints. Orthop Clin North Am. 1987;18(1): 105-114.

32. Qiao YS, Li JK, Shen H, et al. Comparison of arthrodesis and non-fusion to treat Lisfranc injuries. Orthop Surg. 2017;9(1):62-68.

33. Cochran G, Renninger C, Tompane T, Bellamy J, Kuhn K. Primary arthrodesis versus open reduction and internal fixation for low-energy Lisfranc injuries in a young athletic population. Foot Ankle Int 2017;38(9):957-963.

34. Kay RM, Tang CW. Pediatric foot fractures: evaluation and treatment. J Am Acad Orthop Surg. 2001;9(5):308-319.

35. Crawford AH. Fractures and dislocations of the foot and ankle. In: Green NE, Swiontkowski MF, editors. Skeletal Trauma in Children. Philadelphia, PA: WB Saunders; 1994:449-516.

36. Petrisor BA, Ekrol I, Court-Brown C. The epidemiology of metatarsal fractures. Foot Ankle Int. 2006;27(3):172-174.

37. Pao DG, Keats TE, Dussault RG. Avulsion fracture of the base of the fifth metatarsal not seen on conventional radiography of the foot: the need for an additional projection. AJR Am J Roentgenol. 2000;175(2): $549-552$.

38. Lawrence SJ, Botte MJ. Jones' fractures and related fractures of the proximal fifth metatarsal. Foot Ankle. 1993;14(6):358-365.

39. Torg JS, Balduini FC, Zelko RR, Pavlov H, Peff TC, Das M. Fractures of the base of the fifth metatarsal distal to the tuberosity. Classification and guidelines for non-surgical and surgical management. J Bone Joint Surg Am. 1984;66(2):209-214. 
40. Zwitser EW, Breederveld RS. Fractures of the fifth metatarsal; diagnosis and treatment. Injury. 2010;41(6):555-562.

41. Josefsson PO, Karlsson M, Redlund-Johnell I, Wendeberg B. Closed treatment of Jones fracture. Good results in 40 cases after 11-26 years. Acta Orthop Scand. 1994;65(5):545-547.

42. Quill GE Jr. Fractures of the proximal fifth metatarsal. Orthop Clin North Am. 1995;26(2):353-361.

43. Mologne TS, Lundeen JM, Clapper MF, O’Brien TJ. Early screw fixation versus casting in the treatment of acute Jones fractures. Am J Sports Med. 2005;33(7):970-975.

44. Afolayan JO, Dinneen A, Sakellariou A. Tarsal coalitions - what you need to know. Orthop Trauma. 2016;30(1):30-40.

45. Kernbach KJ. Tarsal coalitions: etiology, diagnosis, imaging, and stigmata. Clin Podiatr Med Surg. 2010;27(1):105-117.

46. Stormont DM, Peterson HA. The relative incidence of tarsal coalition. Clin Orthop Relat Res. 1983;(181):28-36.

47. Jayakumar S, Cowell HR. Rigid flatfoot. Clin Orthop Relat Res. 1977;(122):77-84.

48. Sakellariou A, Sallomi D, Janzen DL, Munk PL, Claridge RJ, Kiri VA. Talocalcaneal coalition. Diagnosis with the C-sign on lateral radiographs of the ankle. J Bone Joint Surg Br. 2000;82(4):574-578.

49. Swensen SJ, Otsuka NY. Tarsal coalitions - calcaneonavicular coalitions. Foot Ankle Clin. 2015;20(4):669-679.

50. Mosca VS. Tarsal coalition. In: Weinstein SL, Flynn JM, editors. Lovell and Winter's Pediatric Orthopaedics. 7th ed. Philadelphia, PA: Lippincott Williams \& Wilkins; 2014:1504-1515.

51. Comfort TK, Johnson LO. Resection for symptomatic talocalcaneal coalition. J Pediatr Orthop. 1998;18(3):283-288.

52. Wilde PH, Torode IP, Dickens DR, Cole WG. Resection for symptomatic talocalcaneal coalition. J Bone Joint Surg Br. 1994;76(5):797-801.

53. Aibinder WR, Young EY, Milbrandt TA. Intraoperative threedimensional navigation for talocalcaneal coalition resection. J Foot Ankle Surg. 2017;56(5):1091-1094.

54. Mosca VS, Bevan WP. Talocalcaneal tarsal coalitions and the calcaneal lengthening osteotomy: the role of deformity correction. J Bone Joint Surg Am. 2012;94(17):1584-1594.

55. Lisella JM, Bellapianta JM, Manoli A 2nd. Tarsal coalition resection with pes planovalgus hindfoot reconstruction. J Surg Orthop Adv. 2011;20(2):102-105.

56. Waterman BR, Owens BD, Davey S, Zacchilli MA, Belmont PJ Jr. The epidemiology of ankle sprains in the United States. J Bone Joint Surg Am. 2010;92(13):2279-2284.

57. Shawen SB, Dworak T, Anderson RB. Return to play following ankle sprain and lateral ligament reconstruction. Clin Sports Med. 2016;35(4):697-709.

58. Czajka CM, Tran E, Cai AN, DiPreta JA. Ankle sprains and instability. Med Clin NAm. 2014;98(2):313-329.

59. Kaminski TW, Hertel J, Amendola N, et al. National Athletic Trainers' Association position statement: conservative management and prevention of ankle sprains in athletes. J Athl Train. 2013;48(4):528-545.

60. Maffulli N, Ferran NA. Management of acute and chronic ankle instability. J Am Acad Orthop Surg. 2008;16(10):608-615.
61. Bachmann LM, Kolb E, Koller MT, Steurer J, ter Riet G. Accuracy of Ottawa ankle rules to exclude fractures of the ankle and mid-foot: systematic review. BMJ. 2003;326(7386):417.

62. Dizon JM, Reyes JJ. A systematic review on the effectiveness of external ankle supports in the prevention of inversion ankle sprains among elite and recreational players. J Sci Med Sport. 2010;13(3):309-317.

63. Girard P, Anderson RB, Davis WH, Isear JA, Kiebzak GM. Clinical evaluation of the modified Brostrom-Evans procedure to restore ankle stability. Foot Ankle Int. 1999;20(4):246-252.

64. Yoo JS, Yang EA. Clinical results of an arthroscopic modified Brostrom operation with and without an internal brace. J Orthop Traumatol. 2016;17(4):353-360.

65. Cho BK, Park KJ, Kim SW, Lee HJ, Choi SM. Minimal invasive suture-tape augmentation for chronic ankle instability. Foot Ankle Int. 2015;36(11):1330-1338.

66. Hennrikus WL, Mapes RC, Lyons PM, Lapoint JM. Outcomes of the Chrisman-Snook and modified-Broström procedures for chronic lateral ankle instability. A prospective, randomized comparison. Am J Sports Med. 1996;24(4):400-404.

67. de César PC, Avila EM, de Abreu MR. Comparison of magnetic resonance imaging to physical examination for syndesmotic injury after lateral ankle sprain. Foot Ankle Int. 2011;32(12):1110-1114.

68. Takao M, Ochi M, Oae K, Naito K, Uchio Y. Diagnosis of a tear of the tibiofibular syndesmosis. The role of arthroscopy of the ankle. J Bone Joint Surg Br. 2003;85(3):324-329.

69. Hunt KJ, Phisitkul P, Pirolo J, Amendola A. High ankle sprains and syndesmotic injuries in athletes. J Am Acad Orthop Surg. 2015;23(11):661-673.

70. Moore JA Jr, Shank JR, Morgan SJ, Smith WR. Syndesmosis fixation: a comparison of three and four cortices of screw fixation without hardware removal. Foot Ankle Int. 2006;27(8):567-572.

71. Inge SY, Pull Ter Gunne AF, Aarts CA, Bemelman M. A systematic review on dynamic versus static distal tibiofibular fixation. Injury. 2016;47(12):2627-2634.

72. Rammelt S, Godoy-Santos AL, Schneiders W, Fitze G, Zwipp H. Foot and ankle fractures during childhood: review of the literature and scientific evidence for appropriate treatment. Rev Bras Ortop. 2016;51(6):630-639.

73. Cass JR, Peterson HA. Salter-Harris Type-IV injuries of the distal tibial epiphyseal growth plate, with emphasis on those involving the medial malleolus. J Bone Joint Surg Am. 1983;65(8):1059-1070.

74. Barmada A, Gaynor T, Mubarak SJ. Premature physeal closure following distal tibia physeal fractures: a new radiographic predictor. $J$ Pediatr Orthop. 2003;23(6):733-739.

75. Sankar WN, Chen J, Kay RM, Skaggs DL. Incidence of occult fracture in children with acute ankle injuries. J Pediatr Orthop. 2008;28(5): 500-501.

76. Wuerz TH, Gurd DP. Pediatric physeal ankle fracture. JAm Acad Orthop Surg. 2013;21(4):234-244.

77. Morris B, Mullen S, Schroeppel P, Vopat B. Open physeal fracture of the distal phalanx of the hallux. Am J Emerg Med. 2017;35(7):1035.e-1035.e3.

78. Kensinger DR, Guille JT, Horn BD, Herman MJ. The stubbed great toe: importance of early recognition and treatment of open fractures of the distal phalanx. J Pediatr Orthop. 2001;21(1):31-34.
Orthopedic Research and Reviews

\section{Publish your work in this journal}

Orthopedic Research and Reviews is an international, peer-reviewed, open access journal that focusing on the patho-physiology of the musculoskeletal system, trauma, surgery and other corrective interventions to restore mobility and function. Advances in new technologies, materials, techniques and pharmacological agents are particularly

\section{Dovepress}

welcome. The manuscript management system is completely online and includes a very quick and fair peer-review system, which is al easy to use. Visit http://www.dovepress.com/testimonials.php to read real quotes from published authors. 